\title{
The NICHE Array: Status and Plans
}

\section{Douglas R Bergman*}

The University of Utah

E-mail: bergmanephysics . utah. edu

\section{John F Krizmanic}

Universities Space Research Association

E-mail: jkrizmanicausra.edu

\section{Yoshiki Tsunesada}

Tokyo Institute of Technology

E-mail: tsunesada@cr.phys.titech.jp

The Non-Imaging CHErenkov Array (NICHE) will be a low energy extension to Telescope Array and TALE using an array of closely spaced (100-400 m) light collectors covering an area of up to 2 square $\mathrm{km}$ for the full array. It will be deployed in the field of view of TALE and will overlap with it in energy range. Showers with energies $1-100 \mathrm{PeV}$ will be reconstructed using both the Cherenkov light Lateral Distribution and the Cherenkov Time Width Lateral Distribution. These two methods will allow shower energy and $X_{\max }$ to be determined. A prototype of the array, called $\mathrm{j}$-NICHE, is currently being built and will be deployed in the near future. The design and plans for the full array are presented along with a plan to deploy the counters to perform Cherenkov-hybrid air shower observations.

The 34th International Cosmic Ray Conference,

30 July- 6 August, 2015

The Hague, The Netherlands

${ }^{*}$ Speaker. 


\section{Scientific Motivation}

Measurement of the changing nuclear composition of high-energy Cosmic Rays (CR) at energies around the Knee, $\gtrsim 1 \mathrm{PeV}$, provides a unique tool for understanding the evolution of the high-energy end of the galactic CR spectrum while providing a firm foundation for understanding the composition and spectrum of extragalactic ultra high-energy CRs. However, the current understanding is muddled due to uncertainties inherent to the measurement techniques and/or dependence on hadronic Monte Carlo simulation models required to interpret the data[1]. This ambiguity makes it difficult to confirm the widely adopted hypothesis that the Knee is the result of a Peters cycle[2] due to rigidity-dependent cutoffs of the various galactic CR nuclear components.

One way to minimize this state of uncertainty is to perform a coordinated set of experiments to observe the composition of CRs over many orders-of-magnitude, using techniques that are directly sensitive to composition-dependent observables. One such sensitive observable for measuring $\mathrm{CR}$ composition in air showers is the depth of shower maximum, $X_{\max }$, which is measurable in Fluorescence Telescopes (FTs), Imaging Air-Cherenkov Telescopes (IACTs), and non-imaging Cherenkov Detectors (CDs). Historically, non-imaging CDs and FTs have not been used together to simultaneously measure air showers. However, with the newly demonstrated ability of FTs to work as IACTs when pointed at larger elevation angles[3], it has become possible to combine FTs (as IACTs) and CDs in a hybrid array. In addition, by using both the lateral distribution of Cherenkov photons and the time-domain structure of their arrival times, the spacing of Cherenkov counters can be sufficiently increased to lead to a large enough aperture to overlap with lower-energy FTs (qua FTs) such as TALE. Thus, the measurement of the air-Cherenkov signal on the ground, calibrated with air fluorescence and imaging air-Cherenkov measurements, offers a methodology to provide an accurate measurement of the nuclear composition evolution over a large energy range. NICHE will use an array of easily deployable, non-imaging Cherenkov detectors with a variety of spacings to measure the amplitude and time-spread of the air shower Cherenkov signal to extract CR nuclear composition measurements and to cross-calibrate the Cherenkov energy and composition measurements with that from TA/TALE fluorescence and surface detectors[4].

\section{The Non-imaging Cherenkov Technique}

Energetic electrons generated in an extended air shower (EAS) produce Cherenkov radiation if they move faster than the speed-of-light in the local medium. The index of refraction in the atmosphere increases with depth, yielding Cherenkov cones from altitudes 8-20 km to overlap in a ring of radius $120-140 \mathrm{~m}$ at the ground. Since the interior of the ring is filled by the portion of the air shower at lowest altitudes, showers that develop deeper in the atmosphere will have a larger interior-to-ring ratio. Electron transverse momentum smears the Cherenkov ring on the ground, but the inside-to-outside ratio remains composition, i.e. $X_{\max }$, dependent.

At a given point on the ground, a counter will observe two components of Cherenkov light: one from the bulk of the shower, where some fraction of the transverse electrons are pointing their Cherenkov cones at the counter; and another part due to the smaller portion of the shower where the shower core Cherenkov cone intercepts the counter. Photons in the former component arrive over a long time span because the measurement samples a large portion of the developing air shower, 
while the latter component is narrow in time. When there is no dominant core component observed in a counter, the FWHM can be quite wide. Thus the FWHM in time provides a measure of shower development: deeper showers will have more Cherenkov light coming late due to sampling the air shower over a long path length.

A number of experiments have employed the non-imaging Cherenkov technique (AIROBICC, BLANCA, CACTI, and Tunka) using the Cherenkov Light Distribution (CLD) to measure the CR spectrum, while two experiments (Tunka \& BASJE) have employed the Cherenkov Time Width Lateral distribution technique (references are listed in [5]). The innovation of NICHE is to combine these two techniques to construct an array of sufficiently large area to have significant overlap with TALE air-Cherenkov measurements for $E \gtrsim 1 \mathrm{PeV}$ and TA/TALE air fluorescence measurements for $E \gtrsim 100 \mathrm{PeV}$, leading to a cross-calibration of the FT, IACT and non-imaging CD measurements.

\section{3. j-NICHE}

A "Kakenhi" Grant by Japan Society for the Promotion of Science (JSPS) has been approved, and a prototype array of $15 \mathrm{CDs}$ is currently being developed. The grant is for four years and began in 2014. The funds from the first year of this grant have been used to purchase photomultiplier tubes; specify and procure an FADC data acquisition system from Brains, Inc. in Japan; purchase batteries and solar panels; and design and procure Winston cones (milled in the Dept. of Physics and Astronomy at the University of Utah). Funds from the second year will allows for the design and manufacture of a detector enclosure (Utah), assembly of the counters and deployment in the field. This array will be called j-NICHE to distinguish it from other NICHE endeavors.

The initial plan for deployment is co-locate counters with the surface detectors of the TALE in-fill array. See Figure 1. This plan will immediately give access to significant overlap between $\mathrm{j}$-NICHE and TALE as a fluorescence detector at energies $\gtrsim 60 \mathrm{PeV}$. Given the advent of TALE as a IACT however, a smaller array with 100-m spacing closer to the TALE FD may be used. This would provide imaging-non-imaging Cherenkov hybrid events above $3 \mathrm{PeV}$. The higher flux at 3 $\mathrm{PeV}$ would more than account for the smaller area covered in going from 400-m to 100-m spacing.

The various components of the j-NICHE counters have mostly been purchased. There will be one 3" PMT per counter, Hamamatsu R6233-100. The collection area will be increased and the angular acceptance defined by the use of a Winston cone of opening half-angle $45^{\circ}$. The Winston cones are made by machining a solid aluminum 4" dowel. The cone is then polished. One of the resulting cones is shown in Figure 2. Power for the counters will be provided by a solar panel/battery system. Data acquisition and triggering will be handled by a 200 MSPS FADC/FPGA unit made by Brains, Inc. in Japan. The front and back of the Brains data acquisition modules is shown in Figure 3.

The housing for the $\mathrm{j}$-NICHE counters is now being designed. It will include a rotating platter with a hole that will serve as a shutter, keeping the PMT and Winston cone safe during the daylight hours. It will also house the batteries, data acquisition and control electronics. Rough schematics are shown in Figure 4. 


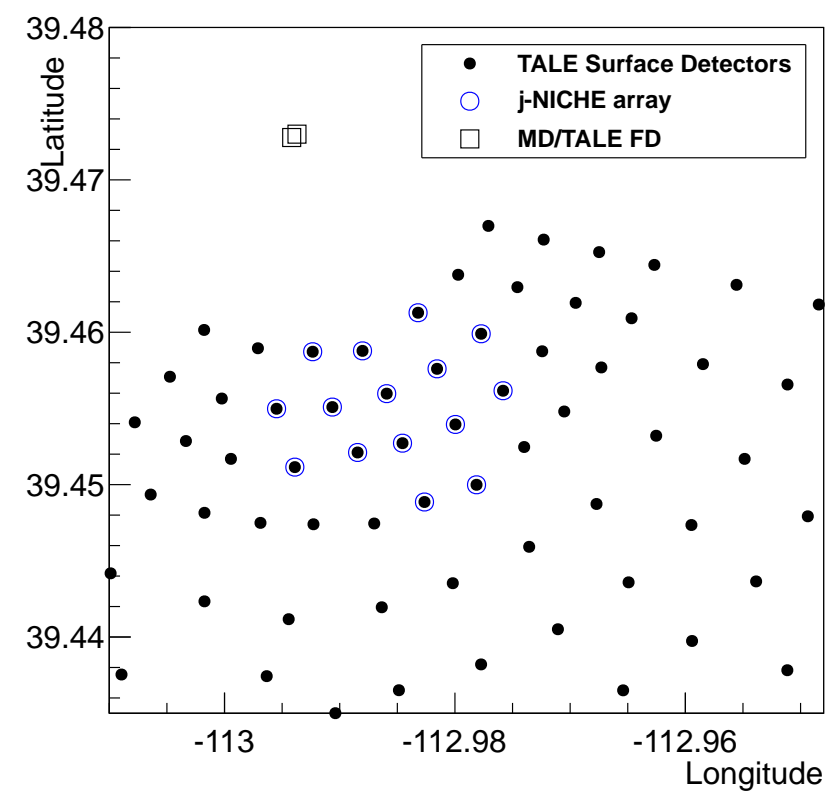

Figure 1: The planned layout of the j-NICHE with respect to the MD/TALE FDs and SDs. Longitude and Latitude are measured in degrees East and degrees North, respectively.

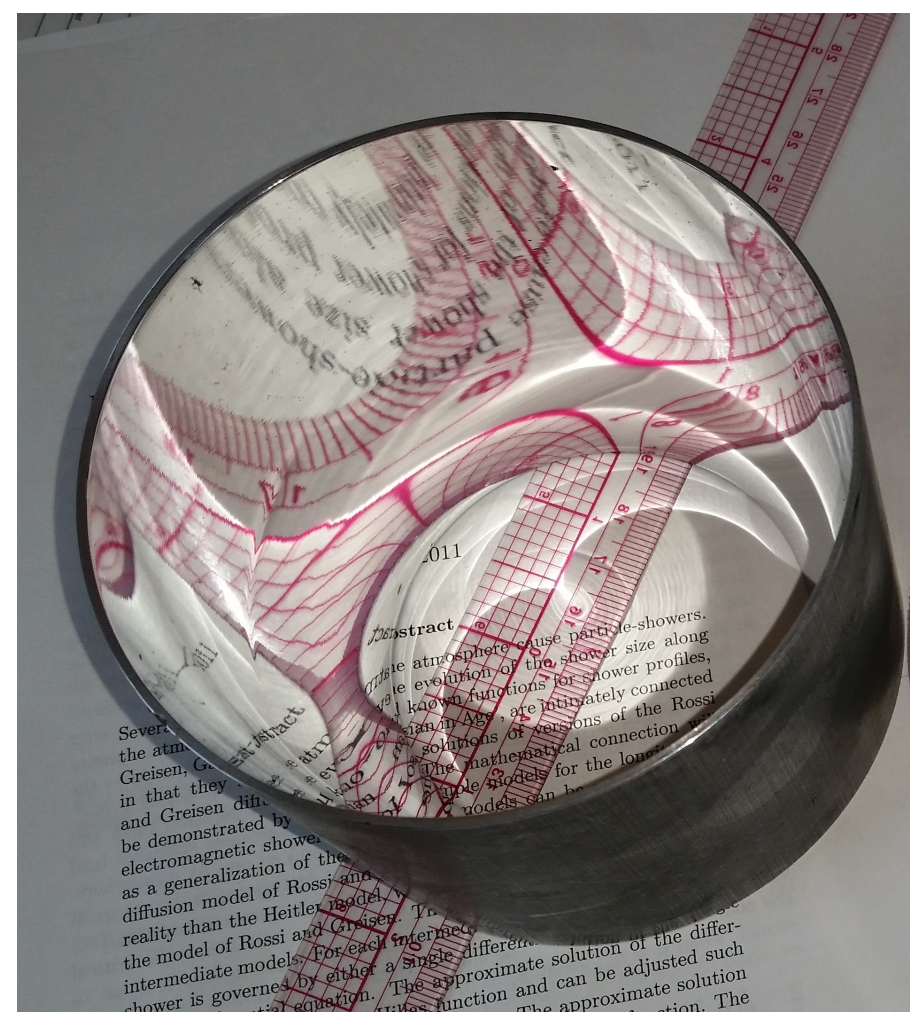

Figure 2: A sample Winston cone. 


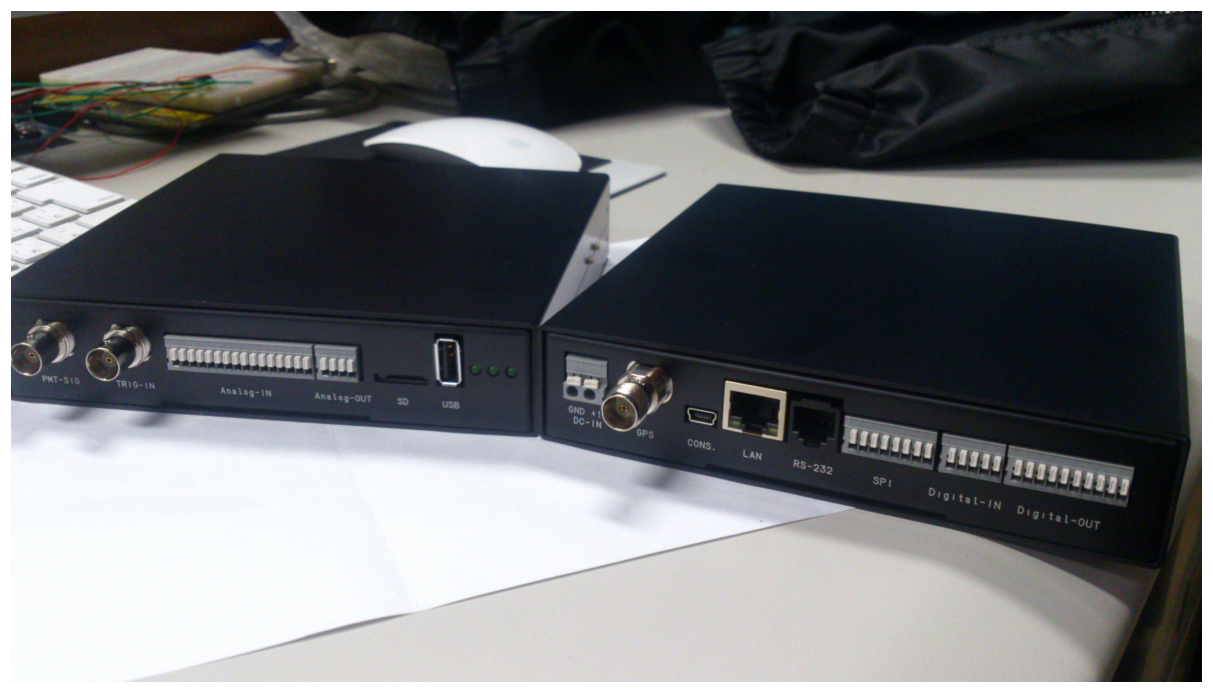

Figure 3: The front and back of the data acquisition models from Brains, Inc.
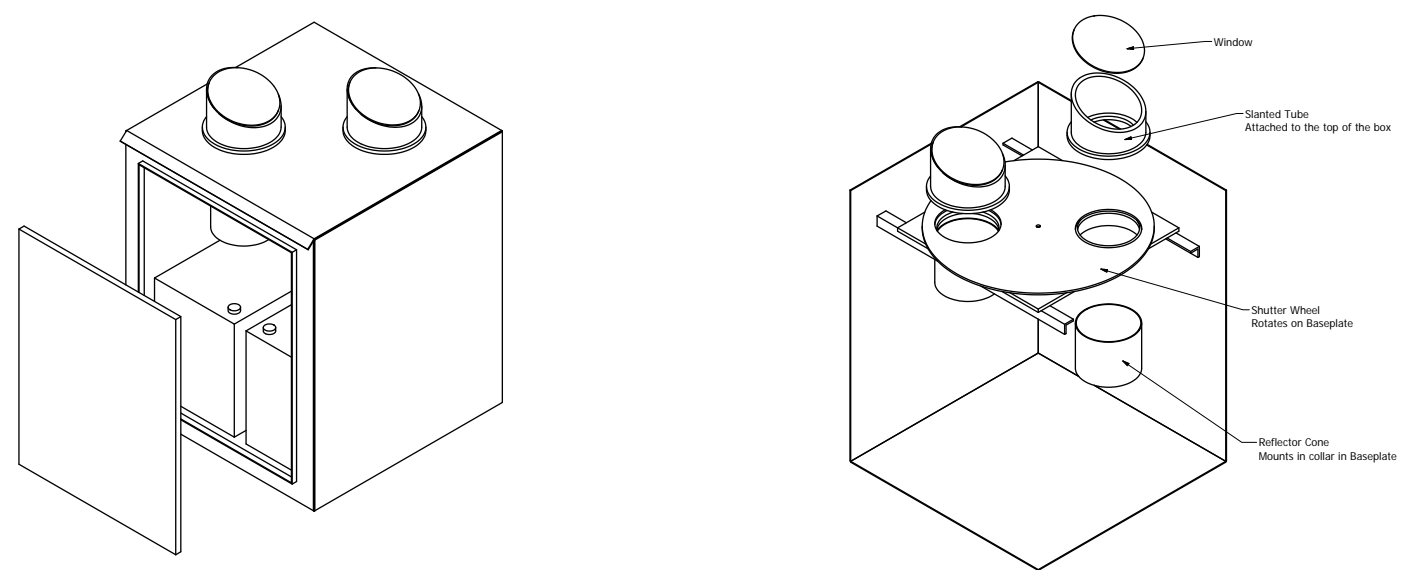

Figure 4: Rough schematic of the counter design. j-NICHE counters would have only one PMT installed per counter. The two-PMT design for the NICHE hybrid array will allow for a local coincidence trigger and thus a lower threshold and/or a lower trigger rate.

\section{100-m Array for Cherenkov Hybrid}

The somewhat surprising ability of the TALE FD to work as an IACT changes the landscape of designing an associated ground array of non-imaging CDs. Fluorescence dominated detection of the TALE FD has a threshold of about $30 \mathrm{PeV}$, and the typical core distances for these events range from $2-5 \mathrm{~km}$. To get a significant overlap with fluorescence then dictates a ground array of $1-2 \mathrm{~km}^{2}$. This large area pushes the separations between counters to several hundred meters. However, the track-like Cherenkov event threshold for TALE is about $2 \mathrm{PeV}$. The lower threshold implies a factor of a few hundred times higher flux, and thus a much smaller area is required. In addition, most events hit the ground in the range 400-2000 $\mathrm{m}$ from the TALE FD. To form 

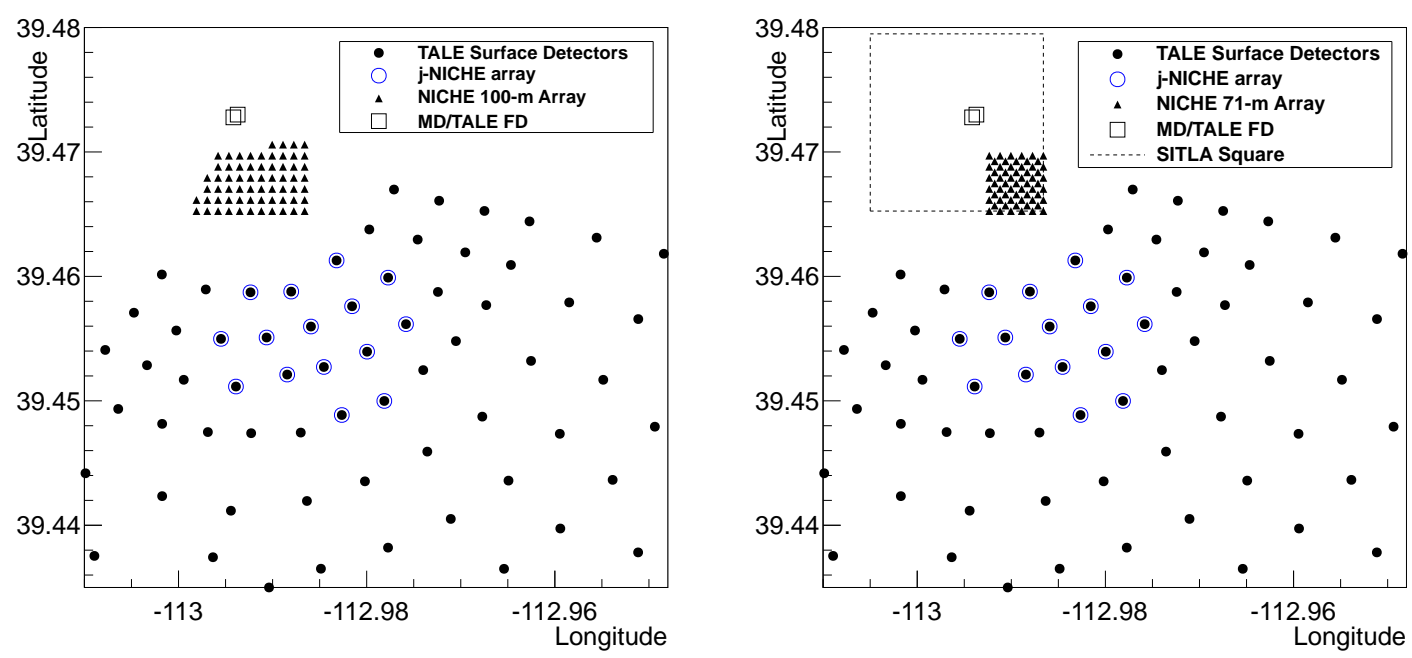

Figure 5: Two possible layouts for NICHE arrays with spacings of $100 \mathrm{~m}$ and $71 \mathrm{~m}$. Longitude and Latitude are measured in degrees East and degrees North, respectively.

a track, the showers must be pointed nearly at the mirrors, but not directly. The viewing angle should be from $1.5-5^{\circ}$. So an array covering some small fraction of a square kilometer, close to the TALE FD is optimal. As it happens, the TALE FD is built close to the center of a square mile of land administered by SITLA (School and Institutional Trust Lands Administration). Deploying detectors on this land has a simpler approval process than deploying on land administered by the BLM (Bureau of Land Management). We are thus planning to build an array of CDs with either 71-m or 100-m spacing within the SITLA square and within the field-of-view of the TALE FD. Two possible layouts are shown in Figure 5.

\section{The Full NICHE Array}

After establishing the air-Cherenkov hybrid method, we will redeploy the NICHE counters from the hybrid array into the TALE SD array, co-siting NICHE counters with TALE SDs where possible. This Array will have significant overlap with TALE in fluorescence at energies above 100 $\mathrm{PeV}$. The proposed layout for this stage of the experiment is shown in Figure 6. This performance of this array is detailed in the poster by J. Krizmanic at this conference[6].

Measurements with the NICHE array in its various configurations, combined with IACT measurements from the TALE FD, and fluorescence and SD measurements from TA will allow the measurement of the CR spectrum and composition over five orders-of-magnitude. Furthermore, all these measurements will be performed by or be calibrated by optical measurements which observe the bulk of the CR-produced extensive air shower.

\section{Acknowledgement}

This work is supported by JSPS Grant-In-Aid for Young Scientists (A) Number 26707011. 


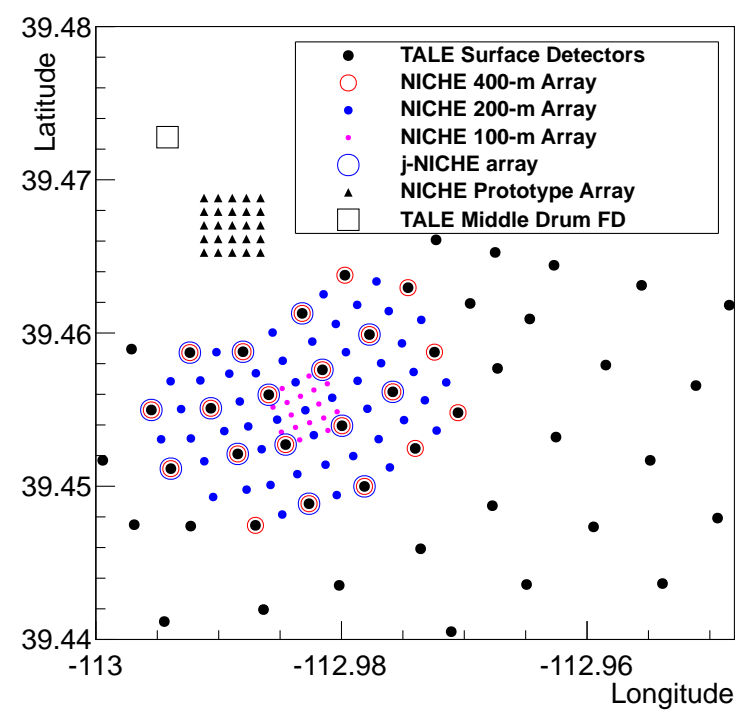

Figure 6: The proposed layout for the full NICHE array. Longitude and Latitude are measured in degrees East and degrees North, respectively.

\section{References}

[1] K.-H. Kampert, M. Unger, Measurements of the cosmic ray composition with air shower experiments, Astropart. Phys. 35 (2012) 660.

[2] B. Peters, Primary energy spectrum and airshowers, Proceedings of the 6th ICRC (Moscow) 3 (1960) 157.

[3] T. Abu-Zayyad, Cosmic Rays Energy Spectrum observed by the TALE detector using Cerenkov light, these proceedings, PoS(ICRC2015)422.

[4] J. Krizmanic, D. Bergman, P. Sokolsky, The Non-Imaging CHErenkov Array (NICHE): A TA/TALE Extension to Measure the Flux and Composition of Very-High Energy Cosmic Rays, Proceedings of the 33rd ICRC (Rio de Janeiro), (2013) 0365.

[5] D. Bergman, J. Krizmanic, NICHE: The non-imaging Cherenkov array, AIP Conf. Proceed. 1516 (2013) 282.

[6] J. Krizmanic, D. Bergman, Y. Tsunesada The Cosmic Ray Nuclear Composition Measurement Performance of the Non-Imaging CHErenkov Array (NICHE), these proceedings,

PoS(ICRC2015)562. 